
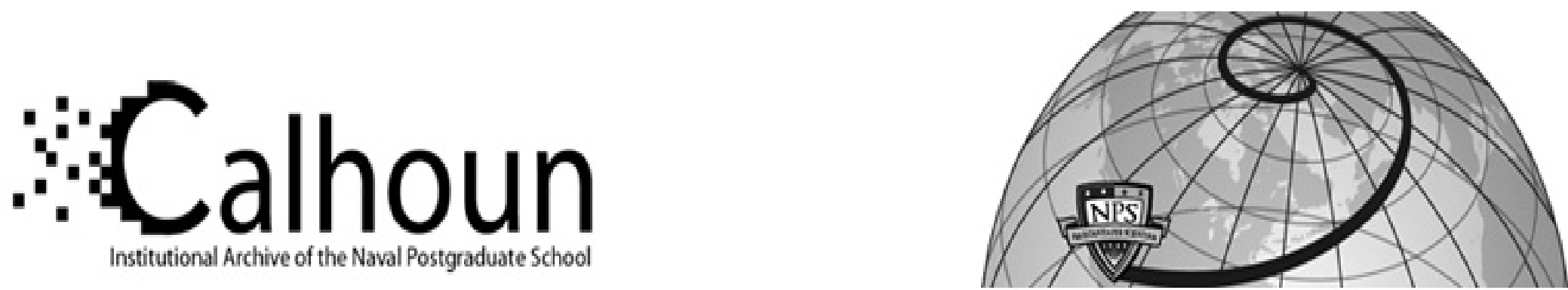

Calhoun: The NPS Institutional Archive DSpace Repository

\title{
Homogeneous flow field effect on the control of Maxwell materials
}

Zhou, Hong; Kang, Wei; Krener, Arthur; Wang, Hongyum

Homogeneous flow field effect on the control of Maxwell materials (with W. Kang, A. Krener and H. Wang), Journal of Non-Newtonian Fluid Mechanics, 150, 104-115, 2008 https://hdl.handle.net/10945/40962

This publication is a work of the U.S. Government as defined in Title 17, United States Code, Section 101. Copyright protection is not available for this work in the United States.

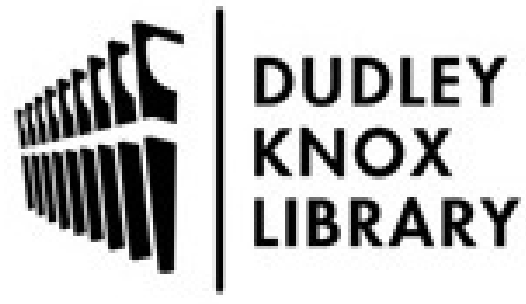

http://www.nps.edu/library
Calhoun is the Naval Postgraduate School's public access digital repository for research materials and institutional publications created by the NPS community. Calhoun is named for Professor of Mathematics Guy K. Calhoun, NPS's first appointed -- and published -- scholarly author.

Dudley Knox Library / Naval Postgraduate School 411 Dyer Road / 1 University Circle Monterey, California USA 93943 


\title{
Homogeneous flow field effect on the control of Maxwell materials
}

\author{
Hong Zhou ${ }^{\mathrm{a}, *}$, Wei Kang ${ }^{\mathrm{a}}$, Arthur Krener ${ }^{\mathrm{a}}$, Hongyun Wang ${ }^{\mathrm{b}}$ \\ ${ }^{a}$ Department of Applied Mathematics, Naval Postgraduate School, 833 Dyer Road, Bldg. 232, SP-250, Monterey, CA 93943-5216, United States \\ ${ }^{\mathrm{b}}$ Department of Applied Mathematics and Statistics, University of California, Santa Cruz, CA 95060, United States
}

Received 16 August 2007; received in revised form 6 October 2007; accepted 24 October 2007

\begin{abstract}
The controllability of viscoelastic fields is a fundamental concept that defines some essential capabilities and limitations of the resulting materials. In this paper, we study the controllability of different homogeneous flow fields of viscoelastic fluids governed by the upper convected Maxwell model. The approach is largely based on the nonlinear geometric control theory. Through the analysis of the control Lie algebra, we find the submanifolds in the state space on which the homogeneous flow fields are weakly controllable. Our approach can be generalized to more complicated systems.
\end{abstract}

(C) 2007 Elsevier B.V. All rights reserved.

Keywords: Upper convected Maxwell model; Controllability; Lie algebra

\section{Introduction}

The controllability of viscoelastic fluids has important applications in the design of desired materials. An important issue of controllability is the question whether it is possible to steer a system to a desirable state with a given set of control inputs.

In [5] the controllability of flows of linear viscoelastic fluids was investigated by Renardy using multi-mode Maxwell models. The state of the system is characterized by the velocities and the viscoelastic stresses; the control input is in the form of the body force. It was found that the system is uncontrollable, unless the initial conditions for the stresses satisfy a set of constraints. For the degenerate case of creeping flow where the density is zero in the equation of motion, there is no controllability unless the control input is distributed along the entire interval in the physical domain. In the presence of inertia, crucial difference occurs between the cases of one or more relaxation modes: For a single relaxation mode (i.e. a Maxwell fluid), exact controllability holds provided the time interval satisfies certain inequality; for multiple relaxation modes, exact controllability holds under modified regularity assumptions. Another piece of work by Renardy [6] was focused on the homogeneous shear flow of viscoelastic fluids with several different constitutive models. For those equations, the state of the system consists of viscoelastic stresses whereas the shear rate is regarded as a control input. For the upper convected Maxwell (UCM) model, it was revealed in [6] that the reachable set, i.e. the states in stress space which are accessible from a given initial condition, is specified by a positive definiteness inequality of the stress tensor. Very recently the result was extended to the control of nonhomogeneous shear flow of an upper convected Maxwell fluid [7] where the state of the system and the available control are the same as those in [5].

The goal of this paper is to extend the work in [6] to a variety of homogeneous flow fields on the control of viscoelastic fluids. Our work differs from [6] in several aspects. First, we include in this study the effect of different homogeneous flow fields in addition to the shear flow addressed in [6]. Secondly, due to its nonlinear nature, our approach is largely based on nonlinear geometric control theory, which is different from the analysis and tools used in [6] for the UCM model under shear flow. The third difference lies in the definition of controllability. Rather than the general concept of controllability, in this paper we adopt a local version of the concept, namely weak controllability. This definition has been widely used in nonlinear control theory as well as in engineering applications.

\footnotetext{
* Corresponding author. Tel.: +1 8316562600.

E-mail address: hzhou@nps.edu (H. Zhou).
} 
In many real life applications of complicated nonlinear systems, engineers prefer to reach a distance target by scheduling a sequence of local movements for the reasons of model uncertainties, system perturbations, and sensor noise. From a theoretical viewpoint, Lie brackets of vector fields provide an efficient tool to prove weak controllability. We note that Lie brackets have been exploited in [6] for a nonlinear model and the Renardy model. But the systems addressed in this paper are different. Among the various types of flows addressed in this paper, the special case of shear flow was also addressed in [6]; and the results are consistent except that we address the concept of weak controllability, not the reachable set derived in [6]. However, for the case of extensional flow, we derive the constraints of the reachable set in Section 4.

Similar to [6], we mostly limit our study to the two-dimensional upper convected Maxwell fluid except for Section 5, in which we introduce some remarks and discussions on three-dimensional flows. However, similar analysis developed in this paper is applicable to higher dimensions (even though more advanced mathematical tools are needed) and other constitutive models.

We organize our paper as follows. First we give a brief discussion on the controllability of control systems. Then we introduce the upper convected Maxwell fluid. After that, we analyze the effect of various homogeneous flow fields on the controllability of Maxwell materials. More precisely, extensional flow, shear flow and general planar linear flow are considered where the submanifold of controllability is characterized for each case. Then we provide a brief discussion on 3D models. Finally, we conclude the paper with a summary of the main results.

\section{The controllability of control systems}

Consider a general nonlinear control system that is affine in control [4]:

$$
\dot{x}=f(x)+\sum_{i=1}^{m} g_{i}(x) u_{i},
$$

where $x$ is the state variable, $u_{i} \in \mathbb{R}, i=1, \ldots, m$, are the control variables. In general, the state variable takes value in a manifold of dimension $n$, denoted by $M$. An important concept for systems defined by (1) is its controllability, which characterizes the ability to maneuver the system from one state to other by proper choice of control. There exists a huge literature on the controllability of control systems. In this paper, we adopt the definition and follow the geometric approach from [2].

Let $x_{0}$ be a point in $M$, the manifold of state variables. A point $x_{1}$ in $M$ is said to be reachable from $x_{0}$ if there exist piecewise continuous input functions, $u_{i}=\alpha_{i}(t)$, so that the trajectory, $x(t)$, of (1) with initial state $x_{0}$ reaches $x_{1}$ in finite time, i.e. $x_{1}=x(T)$ for some $T>0$. For nonlinear control systems, the global reachability is usually very difficult to prove. Instead, a practical solution is to study weak controllability.

Definition 1. A control system is said to be weakly controllable in an open subset $D \subseteq M$ if, for any $x_{0} \in D$, there exists an open neighborhood $U_{0}$ of $x_{0}$ so that the set of points reachable from $x_{0}$ along trajectories inside $U_{0}$ contains at least an open subset of $M$.

If a system is weakly controllable, it implies that the locally reachable states form a "solid" region. More restrictive than weak controllability, the concept of controllability requires that any two points in $M$ are reachable from each other. For linear, time invariant control systems, controllable and weakly controllable are equivalent. However, for nonlinear control systems like those studied in this paper, the determination of controllability requires the global geometric properties of the vector fields in control systems. The description of the set of points reachable from a given point is still an open problem for most control systems. On the other hand, weak controllability can be determined by using the dimension of the control Lie algebra and a distribution generated by the vector fields associated with the control system; nevertheless, weak controllability implies some important properties of a control system.

In (1), $f(x)$ and $g_{i}(x)$ are vector fields on the manifold $M$. Under the Lie bracket operation, $[f, g]$, the space of smooth vector fields on $M$ forms a Lie algebra. This Lie algebra, the smallest subalgebra containing the vector fields $f, g_{1}, \ldots, g_{m}$, is called the control Lie algebra, denoted by $\mathcal{C}$. At each point $x \in M$, the vectors in $\mathcal{C}$ span a vector space. It is denoted by $\Delta_{\mathcal{C}}(x)$, i.e.:

$$
\Delta_{\mathcal{C}}(x)=\operatorname{span}\{X(x) \mid X \text { is a vector field in } \mathcal{C}\} .
$$

This mapping from $M$ to the tangent bundle of $M$ is called a distribution. The following is a useful sufficient condition on the weak controllability of a nonlinear control system.

Definition 2. A control system satisfies the controllability rank condition (CRC) on an open set $D \subset M$ if

$$
\operatorname{dim}\left(\Delta_{\mathcal{C}}(x)\right) \equiv n
$$

for all $x \in D$, where $n$ is the dimension of the manifold $M$.

Theorem 1 (Isidori [2]). A control system of the form (1) is weakly controllable on an open set D if it satisfies the controllability rank condition on $D$. 


\section{The upper convected Maxwell (UCM) model}

The upper convected Maxwell (UCM) model was proposed by J.C. Maxwell over a century ago. It has been widely used in polymer rheology for the description of a viscoelastic fluid under large deformations [1,3]. The UCM model gives a viscoelastic constitutive equation and can be written in the form:

$$
\dot{\mathbf{T}}-(\nabla \mathbf{v}) \mathbf{T}-\mathbf{T}(\nabla \mathbf{v})^{\mathrm{T}}+\lambda \mathbf{T}=2 \mu \mathbf{D},
$$

where $\mathbf{T}$ is the stress tensor, $\mathbf{v}$ the velocity, $\nabla \mathbf{v}$ the velocity gradient tensor, $\lambda$ the relaxation rate, $\mu$ the elastic modulus and $\mathbf{D}$ is the rate-of-deformation tensor (i.e. the symmetric part of the velocity gradient).

We consider 2D homogeneous viscoelastic fluids and denote the stress tensor by

$$
\mathbf{T}=\left[\begin{array}{ll}
T_{11} & T_{12} \\
T_{12} & T_{22}
\end{array}\right],
$$

where $T_{11}$ is the first normal stress difference, $T_{22}$ the second normal stress difference and $T_{12}$ is the shear stress. Suppose the control input is denoted $\dot{\gamma}$ and it is which is closely related to the velocity. Then the general dynamic problem of (3) becomes

$$
\dot{\mathbf{T}}=\mathbf{F}(\dot{\gamma}(t), \mathbf{T}), \quad \mathbf{T}(0)=\mathbf{T}_{0}, \quad \mathbf{T}\left(t_{\text {final }}\right)=\mathbf{T}_{1},
$$

where $\mathbf{T}_{0}$ and $\mathbf{T}_{1}$ are the given initial and final states, respectively. The state of the system (5) is characterized by viscoelastic stress $\mathbf{T}$ with three components $T_{11}, T_{22}$ and $T_{12}$.

\section{Flow field effect on the controllability of Maxwell materials}

Our primary purpose in this section is to study the effect of flow fields on the controllability of Maxwell materials for various types of flows. A weak controllability condition is presented for each case.

\subsection{Extensional flow}

For a fluid in a homogeneous extensional flow with rate $\dot{\gamma}(t)$, the velocity is

$$
\mathbf{v}=\left(\dot{\gamma}(t) \frac{x}{2},-\dot{\gamma}(t) \frac{y}{2}\right),
$$

so the velocity gradient is

$$
\nabla \mathbf{v}=\left[\begin{array}{cc}
\frac{\dot{\gamma}(t)}{2} & 0 \\
0 & -\frac{\dot{\gamma}(t)}{2}
\end{array}\right]
$$

The rate-of-strain tensor becomes

$$
\mathbf{D}=\frac{1}{2}\left[\nabla \mathbf{v}+(\nabla \mathbf{v})^{\mathrm{T}}\right]=\frac{\dot{\gamma}(t)}{2}\left[\begin{array}{cc}
1 & 0 \\
0 & -1
\end{array}\right] .
$$

In component form, (3) becomes

$$
\dot{T}_{11}-(\dot{\gamma}(t)-\lambda) T_{11}=\mu \dot{\gamma}(t), \quad \dot{T}_{12}+\lambda T_{12}=0, \quad \dot{T}_{22}+(\dot{\gamma}(t)+\lambda) T_{22}=-\mu \dot{\gamma}(t) .
$$

Note that $T_{12}$ can be solved exactly:

$$
T_{12}=T_{12}(0) \exp (-\lambda t)
$$

Since the behavior of $T_{12}$ is unaffected by the control, the UCM model (3) is not weakly controllable and hence does not satisfy the $\mathrm{CRC}$ anywhere. However, the state space has a stable and control invariant subspace $T_{12}=0$. All trajectories of the system under any control input asymptotically approach this subspace. Therefore, the ultimate behavior of the control system is represented by the reduced system on the stable subspace:

$$
\dot{T}_{11}-(\dot{\gamma}(t)-\lambda) T_{11}=\mu \dot{\gamma}(t), \quad \dot{T}_{22}+(\dot{\gamma}(t)+\lambda) T_{22}=-\mu \dot{\gamma}(t) .
$$


For mathematical convenience, we introduce

$$
\vec{x}=\left[\begin{array}{l}
x_{1} \\
x_{2}
\end{array}\right]=\left[\begin{array}{l}
T_{11} \\
T_{22}
\end{array}\right]
$$

Then the system (11) can be rewritten as

$$
\frac{\mathrm{d} \vec{x}}{\mathrm{~d} t}=\vec{f}(\vec{x})+\vec{g}(\vec{x}) u
$$

where

$$
u=\dot{\gamma}(t), \quad \vec{f}(\vec{x})=\left[\begin{array}{c}
-\lambda x_{1} \\
-\lambda x_{2}
\end{array}\right], \quad \vec{g}(\vec{x})=\left[\begin{array}{c}
\mu+x_{1} \\
-\mu-x_{2}
\end{array}\right] .
$$

The Lie bracket is

$$
[\vec{f}, \vec{g}]=\nabla \vec{g} \cdot \vec{f}-\nabla \vec{f} \cdot \vec{g}=\left[\begin{array}{c}
\lambda \mu \\
-\lambda \mu
\end{array}\right]
$$

Then we have

$$
\operatorname{det}[\vec{g},[\vec{f}, \vec{g}]]=\operatorname{det}\left[\begin{array}{cc}
\mu+x_{1} & \lambda \mu \\
-\mu-x_{2} & -\lambda, \mu
\end{array}\right]=\lambda \mu\left(x_{2}-x_{1}\right) .
$$

It follows that the subsystem (11) of the UCM model under extensional flow satisfies the CRC and hence is weakly controllable on the set of all states where the two normal stress differences are not equal, i.e. $x_{2} \neq x_{1}$ (or $T_{11} \neq T_{22}$ ).

For extensional flows, it is possible to characterize the set of reachable states for the subsystem defined by (11) which is equivalent to

$$
\dot{T}_{11}=-(\lambda-\dot{\gamma}(t)) T_{11}+\mu \dot{\gamma}(t), \quad \dot{T}_{22}=-(\lambda+\dot{\gamma}(t)) T_{22}-\mu \dot{\gamma}(t) .
$$

Our approach below is inspired by Renardy's work [6].

Introducing new unknown functions:

$$
x=T_{11}+\mu, \quad y=T_{22}+\mu,
$$

the ODE system (17) becomes

$$
\dot{x}=-(\lambda-\dot{\gamma}(t)) x+\lambda \mu, \quad \dot{y}=-(\lambda+\dot{\gamma}(t)) y+\lambda \mu .
$$

If we rescale all the variables as follows:

$$
\tilde{x}=\frac{x}{\mu}, \quad \tilde{y}=\frac{y}{\mu}, \quad \tilde{t}=\lambda t, \quad \tilde{\beta}(\tilde{t})=\frac{\dot{\gamma}(t)}{\lambda},
$$

then after dropping all tildes for simplicity, (18) has the form

$$
\dot{x}=-(1-\beta(t)) x+1, \quad \dot{y}=-(1+\beta(t)) y+1 .
$$

We shall prove the following theorem.

Theorem 2. Let $x(t)$ and $y(t)$ be solutions of the system (19) with initial conditions $x(0)$ and $y(0)$. If $x(0)>0$, then $x(t)>0$ for $t>0$; similarly, if $y(0)>0$, then $y(t)>0$ for $t>0$.

Proof. We argue by contradiction. Suppose $x(\tau)=0$ for some $\tau>0$. Let

$$
t_{1}=\inf \{\tau \mid x(\tau)=0, \tau>0\}
$$

Since we assume that there exists $\tau>0$ such that $x(\tau)=0$, it follows that $t_{1}$ is well defined and is finite. Because $x(t)$ is a continuous function of $t$, we have $x\left(t_{1}\right)=0$. Using the assumption $x(0)>0$ and using the definition of $t_{1}$, we get $x(t)>0$ for $0<t<t_{1}$. Now we evaluate the derivative of $x(t)$ at $t_{1}$ from the first equation of (19) to obtain:

$$
\left.\frac{\mathrm{d} x}{\mathrm{~d} t}\right|_{t=t_{1}}=-\left(1-\beta\left(t_{1}\right)\right) x\left(t_{1}\right)+1=1>0 .
$$


Thus, we have $x\left(t_{1}-\varepsilon\right)<0$ for $\varepsilon$ positive and small enough. This contradicts with the result obtained earlier that $x(t)>0$ for $0<t<t_{1}$. Therefore, we must have $x(t)>0$ for $t>0$. Similar arguments lead to the conclusion that $y(t)$ remains positive for $t>0$ if its initial value is positive.

It is worthwhile to point out that the zero stresses $T_{11}=T_{22}=0$ correspond to $x=y=1$.

From now on, we shall confine our attention to the case where $x(0)>0$ and $y(0)>0$. To find the reachable set, we start with two lemmas.

Lemma 1. The solutions $x(t)$ and $y(t)$ of the system (19) satisfy

$$
\frac{\mathrm{d}(x y)}{\mathrm{d} t}=x+y-2 x y .
$$

Proof. The proof is straightforward. Using (19), we have

$$
\frac{\mathrm{d}(x y)}{\mathrm{d} t}=\frac{\mathrm{d} x}{\mathrm{~d} t} y+x \frac{\mathrm{d} y}{\mathrm{~d} t}=-(1-\beta(t)) x y+y-(1+\beta(t)) x y+x=x+y-2 x y .
$$

Lemma 2. The solutions $x(t)$ and $y(t)$ of the system (19) satisfy

$$
\frac{\mathrm{d} \ln (y / x)}{\mathrm{d} t}=-2 \beta(t)+\frac{1}{y}-\frac{1}{x} .
$$

Proof. Applying the chain rule and (19), we find

$$
\frac{\mathrm{d} \ln x}{\mathrm{~d} t}=\frac{1}{x} \frac{\mathrm{d} x}{\mathrm{~d} t}=-[1-\beta(t)]+\frac{1}{x}, \quad \frac{\mathrm{d} \ln y}{\mathrm{~d} t}=\frac{1}{y} \frac{\mathrm{d} y}{\mathrm{~d} t}=-[1+\beta(t)]+\frac{1}{y} .
$$

It follows immediately that

$$
\frac{\mathrm{d} \ln (y / x)}{d t}=\frac{d(\ln y-\ln x)}{d t}=-2 \beta(t)+\frac{1}{y}-\frac{1}{x} .
$$

Let us introduce two new unknown functions:

$$
\xi=\sqrt{x y}, \quad \eta=\ln \sqrt{\frac{y}{x}} .
$$

Using Eqs. (20) and (21), we find

$$
\begin{aligned}
\frac{\mathrm{d} \sqrt{x y}}{\mathrm{~d} t} & =\frac{1}{2 \sqrt{x y}} \frac{\mathrm{d}(x y)}{\mathrm{d} t}=\frac{1}{2 \sqrt{x y}}(x+y-2 x y)=\frac{1}{2}\left(\sqrt{\frac{x}{y}}+\sqrt{\frac{y}{x}}\right)-\sqrt{x y}, \\
\frac{\mathrm{d} \ln \sqrt{y / x}}{\mathrm{~d} t} & =\frac{1}{2} \frac{\mathrm{d} \ln (y / x)}{\mathrm{d} t}=-\beta(t)+\frac{1}{2}\left(\frac{1}{y}-\frac{1}{x}\right)=-\beta(t)-\frac{1}{\sqrt{x y}} \frac{1}{2}\left(\sqrt{\frac{y}{x}}-\sqrt{\frac{x}{y}}\right) .
\end{aligned}
$$

In terms of $\xi$ and $\eta$, the above ODE system becomes

$$
\frac{\mathrm{d} \xi}{\mathrm{d} t}=\cosh \eta-\xi, \quad \frac{\mathrm{d} \eta}{\mathrm{d} t}=-\beta(t)-\frac{1}{\xi} \sinh \eta .
$$

Note that the system (23) is nonlinear. Furthermore, the evolution of $\xi$ is completely determined by $(\xi, \eta)$ whereas the evolution of $\eta$ depends on the control parameter $\beta(t)$. Of course, $\xi$ is controlled by $\beta(t)$ via its effect on $\eta$.

Theorem 3. For $t>0$, the solution $\xi(t)$ of (23) satisfies

$$
\xi(t)-1 \geq \mathrm{e}^{-t}[\xi(0)-1]
$$

with equality possible only if $\eta(s)=0$ throughout the interval $0 \leq s \leq t$.

Proof. From the first equation of (23), we obtain

$$
\frac{\mathrm{d}(\xi-1)}{\mathrm{d} t}+(\xi-1)=\cosh \eta-1
$$

which gives

$$
\frac{\mathrm{d}\left[\mathrm{e}^{t}(\xi-1)\right]}{\mathrm{d} t}=\mathrm{e}^{t}(\cosh \eta-1) .
$$


Integration from 0 to $t$ yields

$$
\xi(t)-1=\mathrm{e}^{-t}[\xi(0)-1]+\mathrm{e}^{-t} \int_{0}^{t} \mathrm{e}^{s}[\cosh \eta(s)-1] \mathrm{d} s .
$$

Since $\cosh \eta(s) \geq 1$, it follows that

$$
\xi(t)-1 \geq \mathrm{e}^{-t}[\xi(0)-1] \text { for } t>0 .
$$

The equality holds only when $\cosh \eta(s)=1$ for $0 \leq s \leq t$, which is equivalent to $\eta(s)=0$ for $0 \leq s \leq t$. This completes the proof of Theorem 3.

Let $\left(\xi_{0}, \eta_{0}\right)=(\xi(0), \eta(0))$ denote the initial condition and let $\left(\xi_{f}, \eta_{f}\right)$ denote the state we would like to reach at time $t_{f}$. The reachable set at time $t_{f}$ will be described by the following theorem.

\section{Theorem 4.}

(1) If $\xi_{f}-1<\mathrm{e}^{-t_{f}}\left(\xi_{0}-1\right)$, then $\left(\xi_{f}, \eta_{f}\right)$ is not reachable at time $t_{f}$.

(2) If $\xi_{f}-1=\mathrm{e}^{-t_{f}}\left(\xi_{0}-1\right)$, then $\left(\xi_{f}, \eta_{f}\right)$ is reachable at time $t_{f}$ if and only if $\eta_{0}=\eta_{f}=0$.

(3) If $\xi_{f}-1>\mathrm{e}^{-t_{f}}\left(\xi_{0}-1\right)$, then $\left(\xi_{f}, \eta_{f}\right)$ is reachable at time $t_{f}$.

\section{Proof.}

(1) The proof follows directly from Theorem 2.

(2) If $\left(\xi_{f}, \eta_{f}\right)$ is reachable at time $t_{f}$ and $\xi_{f}-1=\mathrm{e}^{-t_{f}}\left(\xi_{0}-1\right)$, then Theorem 2 implies that $\eta(s)=0$ for $0 \leq s \leq t$. Consequently, we have $\eta(0)=\eta\left(t_{f}\right)=0$. That is, $\eta_{0}=\eta_{f}=0$.

On the other hand, if $\eta_{0}=\eta_{f}=0$, we select $\beta(t)=0$ to keep $\eta(t)=0$. This leads to $\xi(t)-1=\mathrm{e}^{-t}\left(\xi_{0}-1\right)$ and thereby $\xi\left(t_{f}\right)=\xi_{f}$. Thus, $\left(\xi_{f}, \eta_{f}\right)$ is reachable at time $t_{f}$.

(3) The proof of (3) needs a lemma.

Lemma 3. Consider solving the ODE system (23) forward in time. Let us select $\beta(t)$ to change $\eta$ linearly in time from $\eta=q_{1}$ at $t=0$ to $\eta=q_{2}$ at $t=\Delta t$. Specifically, we enforce $\mathrm{d} \eta / \mathrm{d} t=\left(q_{2}-q_{1}\right) / \Delta t$ by selecting

$$
\beta(t)=-\frac{1}{\xi(t)} \sinh \eta(t)-\frac{q_{2}-q_{1}}{\Delta t} .
$$

For this choice of $\beta(t)$, it is true that

$$
\mathrm{e}^{-\Delta t}[\xi(0)-1]+\left(1-\mathrm{e}^{-\Delta t}\right)\left(\cosh q_{\min }-1\right) \leq \xi(\Delta t)-1 \leq \mathrm{e}^{-\Delta t}[\xi(0)-1]+\left(\cosh q_{\max }-1\right) \Delta t,
$$

where

$$
q_{\min }=\inf \left\{\mid q \| q \text { is between } q_{1} \text { and } q_{2}\right\}, \quad q_{\max }=\sup \left\{\mid q \| q \text { is between } q_{1} \text { and } q_{2}\right\} .
$$

For the proof of this lemma, we integrate the first equation of (23) to obtain

$$
\xi(\Delta t)-1=\mathrm{e}^{-\Delta t}[\xi(0)-1]+\mathrm{e}^{-\Delta t} \int_{0}^{\Delta t} \mathrm{e}^{s}[\cosh \eta(s)-1] \mathrm{d} s .
$$

Since $q_{\min } \leq|\eta(t)| \leq q_{\max }$ for $0 \leq t \leq \Delta t$, we get $\cosh q_{\min } \leq \cosh \eta(s) \leq \cosh q_{\max }$. Thus, it follows that

$$
\mathrm{e}^{-\Delta t}[\xi(0)-1]+\left(1-\mathrm{e}^{-\Delta t}\right)\left(\cosh q_{\min }-1\right) \leq \xi(\Delta t)-1 \leq \mathrm{e}^{-\Delta t}[\xi(0)-1]+\left(\cosh q_{\max }-1\right) \Delta t .
$$

Now we turn our attention to the proof of part (3). We first select $\beta(t)$ to change $\eta$ linearly in time from $\eta=\eta_{0}$ at $t=0$ to $\eta=q$ at $t=\Delta t$. Then we choose $\beta(t)$ to keep $\eta$ at $\eta=q$ from $t=\Delta t$ to $t=t_{f}-\Delta t$. Finally, we select $\beta(t)$ to change $\eta$ linearly in time from $\eta=q$ at $t=t_{f}-\Delta t$ to $\eta=\eta_{f}$ at $t=t_{f}$. However, the condition $\xi\left(t_{f}\right)=\xi_{f}$ will not be automatically satisfied. Let us consider how to choose $\Delta t$ and $q$ to make $\xi\left(t_{f}\right)=\xi_{f}$.

For the special case of $q=0$, we look at the upper bound on $\xi\left(t_{f}\right)$. Applying Lemma 3 for each of the three time sub-intervals $[0, \Delta t],\left[\Delta t, t_{f}-\Delta t\right]$ and $\left[t_{f}-\Delta t, t_{f}\right]$, we have

$$
\begin{aligned}
& \xi(\Delta t)-1 \leq \mathrm{e}^{-\Delta t}\left(\xi_{0}-1\right)+\left(\cosh \eta_{0}-1\right) \Delta t, \quad \xi\left(t_{f}-\Delta t\right)-1=\mathrm{e}^{-\left(t_{f}-2 \Delta t\right)}[\xi(\Delta t)-1], \\
& \xi\left(t_{f}\right)-1 \leq \mathrm{e}^{-\Delta t}\left[\xi\left(t_{f}-\Delta t\right)-1\right]+\left(\cosh \eta_{f}-1\right) \Delta t .
\end{aligned}
$$


Combining these results, we obtain

$$
\begin{aligned}
\xi\left(t_{f}\right)-1 \leq & \mathrm{e}^{-\Delta t}\left[\xi\left(t_{f}-\Delta t\right)-1\right]+\left(\cosh \eta_{f}-1\right) \Delta t \leq \mathrm{e}^{-\left(t_{f}-\Delta t\right)}[\xi(\Delta t)-1]+\left(\cosh \eta_{f}-1\right) \Delta t \leq \mathrm{e}^{-t_{f}}\left(\xi_{0}-1\right) \\
& +\left(\cosh \eta_{0}-1\right) \Delta t+\left(\cosh \eta_{f}-1\right) \Delta t .
\end{aligned}
$$

Recall that in part (3) we assume $\xi_{f}-1>\mathrm{e}^{-t_{f}}\left(\xi_{0}-1\right)$. So we can select $\Delta t$ small enough such that

$$
\xi\left(t_{f}\right)-1<\xi_{f}-1 \text { for } q=0 .
$$

Once such a $\Delta t$ is found, we fix it. For the general case, we look at the lower bound on $\xi\left(t_{f}\right)$. Applying Lemma 3 for each of the three time sub-intervals $[0, \Delta t],\left[\Delta t, t_{f}-\Delta t\right]$ and $\left[t_{f}-\Delta t, t_{f}\right]$, we get

$$
\begin{aligned}
& \xi(\Delta t)-1 \geq \mathrm{e}^{-\Delta t}\left(\xi_{0}-1\right), \quad \xi\left(t_{f}-\Delta t\right)-1 \geq \mathrm{e}^{-\left(t_{f}-2 \Delta t\right)}[\xi(\Delta t)-1]+\left[1-\mathrm{e}^{-\left(t_{f}-2 \Delta t\right)}\right](\cosh q-1), \\
& \xi\left(t_{f}\right)-1 \geq \mathrm{e}^{-\Delta t}\left[\xi\left(t_{f}-\Delta t\right)-1\right] .
\end{aligned}
$$

Putting all these results together yields

$$
\begin{aligned}
\xi\left(t_{f}\right)-1 & \geq \mathrm{e}^{-\Delta t}\left[\xi\left(t_{f}-\Delta t\right)-1\right] \geq \mathrm{e}^{-\left(t_{f}-\Delta t\right)}[\xi(\Delta t)-1]+\mathrm{e}^{-\Delta t}\left[1-\mathrm{e}^{-\left(t_{f}-2 \Delta t\right)}\right](\cosh q-1) \\
& \geq \mathrm{e}^{-t_{f}}\left(\xi_{0}-1\right)+\mathrm{e}^{-\Delta t}\left[1-\mathrm{e}^{-\left(t_{f}-2 \Delta t\right)}\right](\cosh q-1) .
\end{aligned}
$$

Let us select $q$ large enough such that

$$
\xi\left(t_{f}\right)-1>\xi_{f}-1 \text { for } q \text { sufficiently large. }
$$

Since $\xi\left(t_{f}\right)$ is continuous in $q$, it follows from (24) and (25) that we can find a value of $q$ such that $\xi\left(t_{f}\right)=\xi_{f}$. This concludes the proof of Theorem 4.

In summary, the reachable set is $R+R_{0}$ where $R$ and $R_{0}$ in the $(\xi, \eta)$ plane are

$$
R=\left\{\left(\xi_{f}, \eta_{f}\right) \mid \xi_{f}-1>\mathrm{e}^{-t_{f}}\left(\xi_{0}-1\right)\right\}, \quad R_{0}=\left\{\left(\xi_{f}, \eta_{f}\right) \mid \xi_{f}-1=\mathrm{e}^{-t_{f}}\left(\xi_{0}-1\right) \text { and } \eta_{f}=\eta_{0}=0\right\} .
$$

In the $(x, y)$ plane, $R$ and $R_{0}$ are given by

$$
R=\left\{\left(x_{f}, y_{f}\right) \mid \sqrt{x_{f} y_{f}}-1>\mathrm{e}^{-t_{f}}\left(\sqrt{x_{0} y_{0}}-1\right)\right\}, \quad R_{0}=\left\{\left(x_{f}, y_{f}\right) \mid \sqrt{x_{f} y_{f}}-1=\mathrm{e}^{-t_{f}}\left(\sqrt{x_{0} y_{0}}-1\right) \text { and } \frac{y_{f}}{x_{f}}=\frac{y_{0}}{x_{0}}=1\right\} .
$$

In terms of $\left(T_{11}, T_{22}\right)$ and the original time before scaling, $R$ and $R_{0}$ have the expressions:

$$
\begin{aligned}
R= & \left\{\left(T_{11}\left(t_{f}\right), T_{22}\left(t_{f}\right)\right) \mid \sqrt{\left(\frac{T_{11}\left(t_{f}\right)}{\mu}+1\right)\left(\frac{T_{22}\left(t_{f}\right)}{\mu}+1\right)}-1>\mathrm{e}^{-\lambda t_{f}}\left[\sqrt{\left(\frac{T_{11}(0)}{\mu}+1\right)\left(\frac{T_{22}(0)}{\mu}+1\right)}-1\right]\right\}, \\
R_{0}= & \left\{\left(T_{11}\left(t_{f}\right), T_{22}\left(t_{f}\right)\right) \mid \sqrt{\left(\frac{T_{11}\left(t_{f}\right)}{\mu}+1\right)\left(\frac{T_{22}\left(t_{f}\right)}{\mu}+1\right)}-1=\mathrm{e}^{-\lambda t_{f}}\left[\sqrt{\left(\frac{T_{11}(0)}{\mu}+1\right)\left(\frac{T_{22}(0)}{\mu}+1\right)}-1\right]\right. \\
& \text { and } \left.T_{11}(0)=T_{22}(0), T_{11}\left(t_{f}\right)=T_{22}\left(t_{f}\right)\right\} .
\end{aligned}
$$

\subsection{Shear flow}

For a shear flow with rate $\dot{\gamma}(t)$, the velocity is

$$
\mathbf{v}=(\dot{\gamma}(t) y, 0) .
$$

The rate-of-strain tensor becomes

$$
\mathbf{D}=\frac{1}{2}\left[\nabla \mathbf{v}+(\nabla \mathbf{v})^{\mathrm{T}}\right]=\frac{\dot{\gamma}(t)}{2}\left[\begin{array}{ll}
0 & 1 \\
1 & 0
\end{array}\right] .
$$

The UCM model (3) becomes

$$
\dot{T}_{11}-2 \dot{\gamma}(t) T_{12}+\lambda T_{11}=0, \quad \dot{T}_{12}-\dot{\gamma}(t) T_{22}+\lambda T_{12}=\mu \dot{\gamma}(t), \quad \dot{T}_{22}+\lambda T_{22}=0 .
$$


Then $T_{22}(t)=T_{22}(0) \exp (-\lambda t)$ so the system is not weakly controllable. We consider the subsystem where $T_{22}=0$ :

$$
\dot{T}_{11}-2 \dot{\gamma}(t) T_{12}+\lambda T_{11}=0, \quad \dot{T}_{12}+\lambda T_{12}=\mu \dot{\gamma}(t) .
$$

The system (29) can be expressed as

where $\frac{\mathrm{d} \vec{x}}{\mathrm{~d} t}=\vec{f}(\vec{x})+\vec{g}(\vec{x}) u$,

$$
\vec{x}=\left[\begin{array}{l}
x_{1} \\
x_{2}
\end{array}\right]=\left[\begin{array}{l}
T_{11} \\
T_{12}
\end{array}\right], \quad u=\dot{\gamma}(t), \quad \vec{f}(\vec{x})=\left[\begin{array}{l}
-\lambda x_{1} \\
-\lambda x_{2}
\end{array}\right], \quad \vec{g}(\vec{x})=\left[\begin{array}{c}
2 x_{2} \\
\mu
\end{array}\right] .
$$

The Lie bracket is

So

$$
[\vec{f}, \vec{g}]=\left[\begin{array}{c}
0 \\
\lambda \mu
\end{array}\right]
$$

$$
\operatorname{det}[\vec{g},[\vec{f}, \vec{g}]]=2 \lambda \mu x_{2} \text {. }
$$

Therefore, the subsystem (29) of the UCM model under shear flow satisfies the CRC when the shear stress is nonzero, i.e. $x_{2} \neq 0$ (or $T_{12} \neq 0$ ) and hence is weakly controllable.

By manipulating the system (29), it was found that the set of reachable states of (29) is given precisely by the following inequality [6]:

$$
\mu T_{11}\left(t_{f}\right)-T_{12}\left(t_{f}\right)^{2} \geq \mathrm{e}^{-\lambda t_{f}}\left[\mu T_{11}(0)-T_{12}(0)^{2}\right] .
$$

Note that the condition (34) is stronger than our result in this special case because it gives global reachable sets whereas ours is a condition on local controllability only.

\subsection{General planar linear flow}

Now we consider a general planar linear flow with velocity

$$
\mathbf{v}=\left(p_{1}(t) x+p_{2}(t) y, p_{3}(t) x-p_{1}(t) y\right) .
$$

This general case is different from the previous ones because an invariant subspace may not exist. As a result, we have to deal with the full system model rather than a reduced control system. The rate-of-strain tensor is

$$
\mathbf{D}=\frac{1}{2}\left[\nabla \mathbf{v}+(\nabla \mathbf{v})^{\mathrm{T}}\right]=\left[\begin{array}{cc}
p_{1}(t) & \frac{p_{2}(t)+p_{3}(t)}{2} \\
\frac{p_{2}(t)+p_{3}(t)}{2} & -p_{1}(t)
\end{array}\right] .
$$

Then the UCM model (3) becomes

$$
\begin{aligned}
& \dot{T}_{11}-2\left(p_{1}(t) T_{11}+p_{2}(t) T_{12}\right)+\lambda T_{11}=2 \mu p_{1}(t), \quad \dot{T}_{12}-\left(p_{2}(t) T_{22}+p_{3}(t) T_{11}\right)+\lambda T_{12}=\mu\left(p_{2}(t)+p_{3}(t)\right), \\
& \dot{T}_{22}-2\left(p_{3}(t) T_{12}-p_{1}(t) T_{22}\right)+\lambda T_{22}=-2 \mu p_{1}(t) .
\end{aligned}
$$

The system (37) can be cast into

where $\frac{\mathrm{d} \vec{x}}{\mathrm{~d} t}=\vec{f}(\vec{x})+\vec{g}_{1}(\vec{x}) u_{1}+\vec{g}_{2}(\vec{x}) u_{2}+\vec{g}_{3}(\vec{x}) u_{3}$,

$$
\begin{gathered}
\vec{x}=\left[\begin{array}{l}
x_{1} \\
x_{2} \\
x_{3}
\end{array}\right]=\left[\begin{array}{l}
T_{11} \\
T_{12} \\
T_{22}
\end{array}\right], \quad u_{1}=p_{1}(t), \quad u_{2}=p_{2}(t), \quad u_{3}=p_{3}(t), \quad \vec{f}(\vec{x})=\left[\begin{array}{c}
-\lambda x_{1} \\
-\lambda x_{2} \\
-\lambda x_{3}
\end{array}\right], \\
\vec{g}_{1}(\vec{x})=\left[\begin{array}{c}
2 \mu+2 x_{1} \\
0 \\
-2 \mu-2 x_{3}
\end{array}\right], \quad \vec{g}_{2}(\vec{x})=\left[\begin{array}{c}
2 x_{2} \\
\mu+x_{3} \\
0
\end{array}\right], \quad \vec{g}_{3}(\vec{x})=\left[\begin{array}{c}
0 \\
\mu+x_{1} \\
2 x_{2}
\end{array}\right] .
\end{gathered}
$$

It is practical to consider the situations where only one $p_{i}(t)$ is a control parameter and the other two are constants. Therefore, we consider three cases. 
Case 1. $p_{1}(t)$ is the only control parameter and other parameters are constants.

The system (37) can be rewritten as

$\frac{\mathrm{d} \vec{x}}{\mathrm{~d} t}=\vec{f}_{1}(\vec{x})+\vec{g}_{1}(\vec{x}) u_{1}$,

where $\vec{x}, \vec{g}_{1}, u_{1}$ are defined in (39) and

$\vec{f}_{1}(\vec{x})=\left[\begin{array}{c}-\lambda x_{1}+2 x_{2} p_{2} \\ -\lambda x_{2}+\left(\mu+x_{3}\right) p_{2}+\left(\mu+x_{1}\right) p_{3} \\ -\lambda x_{3}+2 x_{2} p_{3}\end{array}\right]$

After some calculations we obtain

$\left[\vec{g}_{1},\left[\vec{f}_{1}, \vec{g}_{1}\right],\left[\vec{f}_{1},\left[\vec{f}_{1}, \vec{g}_{1}\right]\right]\right]=\left[\begin{array}{ccc}2 \mu+2 x_{1} & 4 x_{2} p_{2}+2 \lambda \mu & 8 p_{2} p_{3}\left(\mu+x_{1}\right)+2 \lambda^{2} \mu \\ 0 & -2 p_{3}\left(\mu+x_{1}\right)+2 p_{2}\left(\mu+x_{3}\right) & 4 \lambda \mu\left(p_{2}-p_{3}\right) \\ -2 \mu-2 x_{3} & -4 x_{2} p_{3}-2 \lambda \mu & -8 p_{2} p_{3}\left(\mu+x_{3}\right)-2 \lambda^{2} \mu\end{array}\right]$,

and

$\operatorname{det}\left(\left[\vec{g}_{1},\left[\vec{f}_{1}, \vec{g}_{1}\right],\left[\vec{f}_{1},\left[\vec{f}_{1}, \vec{g}_{1}\right]\right]\right]\right)=8 \lambda^{2} \mu\left(x_{1}-x_{3}\right)\left(x_{1} p_{3}-x_{3} p_{2}\right)+8 \lambda \mu\left(p_{2}-p_{3}\right)$

$$
\times\left[\lambda \mu\left(x_{1}-x_{3}\right)-4 \mu x_{2}\left(p_{2}-p_{3}\right)+4 x_{2}\left(x_{1} p_{3}-x_{3} p_{2}\right)\right] .
$$

- If $p_{2}=p_{3}$, then the determinant (43) is not zero if $x_{1} \neq x_{3}$, which implies that the CRC is satisfied and the UCM system (37) is weakly controllable there.

- If $p_{2} \neq p_{3}$, we set the determinant (43) to zero and solve for $x_{2}$ in terms of $x_{1}$ and $x_{3}$. After some algebras, we find that

$x_{2}=\frac{\lambda\left(x_{1}-x_{3}\right)\left[\mu\left(p_{2}-p_{3}\right)+\left(x_{1} p_{3}-x_{3} p_{2}\right)\right]}{4\left(p_{2}-p_{3}\right)\left[\mu\left(p_{2}-p_{3}\right)-\left(x_{1} p_{3}-x_{3} p_{2}\right)\right]}$

provided that $\mu\left(p_{2}-p_{3}\right)-\left(x_{1} p_{3}-x_{3} p_{2}\right) \neq 0$. In other words, if $p_{2} \neq p_{3}$ and $\mu\left(p_{2}-p_{3}\right)-\left(x_{1} p_{3}-x_{3} p_{2}\right) \neq 0$, then the determinant (43) does not vanish if $x_{2}$ does not lie on the surface described by (44). As a result, the system (37) satisfies the CRC and is weakly controllable there.

- If $p_{2} \neq p_{3}$ and $\mu\left(p_{2}-p_{3}\right)-\left(x_{1} p_{3}-x_{3} p_{2}\right)=0$, then the determinant (43) simplifies to

$\operatorname{det}\left(\left[\vec{g}_{1},\left[\vec{f}_{1}, \vec{g}_{1}\right],\left[\vec{f}_{1},\left[\vec{f}_{1}, \vec{g}_{1}\right]\right]\right]\right)=16 \lambda^{2} \mu^{2}\left(p_{2}-p_{3}\right)\left(x_{1}-x_{3}\right)$.

So the system (37) satisfies the CRC and is weakly controllable if $x_{1} \neq x_{3}$.

To summarize, we consider the state-parameter space:

$R^{5}=\left\{\left(x_{1}, x_{2}, x_{3}, p_{2}, p_{3}\right) \mid x_{i}, p_{j} \in R\right\}$.

Define some surfaces in $R^{5}$ by

$S_{1}=\left\{\left(x_{1}, x_{2}, x_{3}, p_{2}, p_{3}\right) \mid p_{2}=p_{3}\right\}, \quad S_{2}=\left\{\left(x_{1}, x_{2}, x_{3}, p_{2}, p_{3}\right) \mid \mu\left(p_{2}-p_{3}\right)-\left(x_{1} p_{3}-x_{3} p_{2}\right)=0\right\}$,

$S_{3}=\left\{\left(x_{1}, x_{2}, x_{3}, p_{2}, p_{3}\right) \mid x_{2}=\frac{\lambda\left(x_{1}-x_{3}\right)\left[\mu\left(p_{2}-p_{3}\right)+\left(x_{1} p_{3}-x_{3} p_{2}\right)\right]}{4\left(p_{2}-p_{3}\right)\left[\mu\left(p_{2}-p_{3}\right)-\left(x_{1} p_{3}-x_{3} p_{2}\right)\right]}\right\}, \quad S_{4}=\left\{\left(x_{1}, x_{2}, x_{3}, p_{2}, p_{3}\right) \mid x_{1}=x_{3}\right\}$.

Then in the state-parameter space, $R^{5}$, the system is weakly controllable in $R^{5} \backslash\left\{S_{1} \cup S_{2} \cup S_{3}\right\}$. In $S_{1}$, the system is weakly controllable in $S_{1} \backslash S_{4}$; In $S_{2}$, the system is weakly controllable in $S_{2} \backslash\left\{S_{1} \cup S_{4}\right\}$.

Case 2. $p_{2}(t)$ is the only control parameter.

The system (37) can be rewritten as

$\frac{\mathrm{d} \vec{x}}{\mathrm{~d} t}=\vec{f}_{2}(\vec{x})+\vec{g}_{2}(\vec{x}) u_{2}$, 
where $\vec{x}, \vec{g}_{2}, u_{2}$ are defined in (39) and

$\vec{f}_{2}(\vec{x})=\left[\begin{array}{c}-\lambda x_{1}+2\left(\mu+x_{1}\right) p_{1} \\ -\lambda x_{2}+\left(\mu+x_{1}\right) p_{3} \\ -\lambda x_{3}-2\left(\mu+x_{3}\right) p_{1}+2 x_{2} p_{3}\end{array}\right]$.

Then we have

$\left[\vec{g}_{2},\left[\vec{f}_{2}, \vec{g}_{2}\right],\left[\vec{f}_{2},\left[\vec{f}_{2}, \vec{g}_{2}\right]\right]\right]=\left[\begin{array}{ccc}2 x_{2} & 2\left(x_{1}+\mu\right) p_{3}-4 x_{2} p_{1} & -4 p_{1} p_{3}\left(\mu+x_{1}\right)+2 \lambda \mu p_{3}+8 x_{2} p_{1}^{2} \\ \mu+x_{3} & -2 p_{1}\left(\mu+x_{3}\right)+\lambda \mu & 4 p_{1}^{2}\left(\mu+x_{3}\right)-2 p_{3}^{2}\left(\mu+x_{1}\right)+\lambda \mu\left(\lambda-2 p_{1}\right) \\ 0 & -2 p_{3}\left(\mu+x_{3}\right) & 4 p_{1} p_{3}\left(\mu+x_{3}\right)-4 p_{3}\left(x_{2} p_{3}+\lambda \mu\right)\end{array}\right]$,

and

$\operatorname{det}\left(\left[\vec{g}_{2},\left[\vec{f}_{2}, \vec{g}_{2}\right],\left[\vec{f}_{2},\left[\vec{f}_{2}, \vec{g}_{2}\right]\right]\right]\right)=4 \lambda \mu p_{3}\left[p_{3}\left(\mu^{2}-x_{3}^{2}\right)+2 p_{3} x_{1}\left(\mu+x_{3}\right)+\lambda x_{2}\left(x_{3}-\mu\right)-2 p_{3} x_{2}^{2}\right]$.

If we set the determinant (50) to be zero and solve $x_{1}$ in terms of $x_{2}$ and $x_{3}$, we find that

$x_{1}=\frac{p_{3}\left(2 x_{2}^{2}+x_{3}^{2}-\mu^{2}\right)-\lambda x_{2}\left(x_{3}-\mu\right)}{2 p_{3}\left(\mu+x_{3}\right)}$

provided that the denominator $\mu+x_{3} \neq 0$. From this, we have the following conclusions.

- If $\mu+x_{3} \neq 0$ and $x$ does not lie on the surface given by (51), then the determinant (50) is not zero, the CRC holds and the system (37) is weakly controllable.

- If $\mu+x_{3}=0$, i.e. $x_{3}=-\mu$, then the determinant is reduced to

$\operatorname{det}\left(\left[\vec{g}_{2},\left[\vec{f}_{2}, \vec{g}_{2}\right],\left[\vec{f}_{2},\left[\vec{f}_{2}, \vec{g}_{2}\right]\right]\right]\right)=-8 \lambda \mu p_{3} x_{2}\left(\lambda \mu+p_{3} x_{2}\right)$.

Hence the determinant does not vanish if $x_{2} \neq 0$ and $x_{2} \neq-\lambda \mu / p_{3}$. In this case, the CRC holds and the system (37) is weakly controllable.

Let us summarize our results geometrically. In $R^{5}=\left\{\left(x_{1}, x_{2}, x_{3}, p_{1}, p_{3}\right) \mid x_{i}, p_{j} \in R\right\}$, define three surfaces given by

$S_{5}=\left\{\left(x_{1}, x_{2}, x_{3}, p_{1}, p_{3}\right) \mid \mu+x_{3}=0\right\}, \quad S_{6}=\left\{\left(x_{1}, x_{2}, x_{3}, p_{1}, p_{3}\right) \mid x_{1}=\frac{p_{3}\left(2 x_{2}^{2}+x_{3}^{2}-\mu^{2}\right)-\lambda x_{2}\left(x_{3}-\mu\right)}{2 p_{3}\left(\mu+x_{3}\right)}\right\}$,

$S_{7}=\left\{\left(x_{1}, x_{2}, x_{3}, p_{1}, p_{3}\right) \mid x_{2}=0\right.$ or $\left.\lambda \mu+p_{3} x_{2}=0\right\}$.

Then the system is weakly controllable at all points in $R^{5} \backslash\left\{S_{5} \cup S_{6}\right\}$. In $S_{5}$, the system is weakly controllable at all points in $S_{5} \backslash S_{7}$.

Case 3. $p_{3}(t)$ is the only control parameter.

The system (37) can be rewritten as

$\frac{\mathrm{d} \vec{x}}{\mathrm{~d} t}=\vec{f}_{3}(\vec{x})+\vec{g}_{3}(\vec{x}) u_{3}$,

where $\vec{x}, \vec{g}_{3}, u_{3}$ are defined in (39) and

$\vec{f}_{3}(\vec{x})=\left[\begin{array}{c}-\lambda x_{1}+2\left(\mu+x_{1}\right) p_{1}+2 x_{2} p_{2} \\ -\lambda x_{2}+\left(\mu+x_{3}\right) p_{2} \\ -\lambda x_{3}-2\left(\mu+x_{3}\right) p_{1}\end{array}\right]$.

Then we have

$\left[\vec{g}_{3},\left[\vec{f}_{3}, \vec{g}_{3}\right],\left[\vec{f}_{3},\left[\vec{f}_{3}, \vec{g}_{3}\right]\right]\right]=\left[\begin{array}{ccc}0 & -2\left(x_{1}+\mu\right) p_{2} & -4 p_{1} p_{2}\left(\mu+x_{1}\right)-4 p_{2}\left(x_{2} p_{2}+\lambda \mu\right) \\ \mu+x_{1} & 2 p_{1}\left(\mu+x_{1}\right)+\lambda \mu & 4 p_{1}^{2}\left(\mu+x_{1}\right)-2 p_{2}^{2}\left(\mu+x_{3}\right)+\lambda \mu\left(\lambda+2 p_{1}\right) \\ 2 x_{2} & 2 p_{2}\left(\mu+x_{3}\right)+4 x_{2} p_{1} & 4 p_{1} p_{2}\left(\mu+x_{3}\right)+8 p_{1}^{2} x_{2}+2 p_{2} \lambda \mu\end{array}\right]$,

and

$\operatorname{det}\left(\left[\vec{g}_{3},\left[\vec{f}_{3}, \vec{g}_{3}\right],\left[\vec{f}_{3},\left[\vec{f}_{3}, \vec{g}_{3}\right]\right]\right]\right)=-4 \lambda \mu p_{2}\left[p_{2}\left(\mu^{2}-x_{1}^{2}\right)+2 p_{2} x_{3}\left(\mu+x_{1}\right)+\lambda x_{2}\left(x_{1}-\mu\right)-2 p_{2} x_{2}^{2}\right]$. 
As before, we set the determinant (57) to be zero and solve $x_{3}$ in terms of $x_{1}$ and $x_{2}$ and obtain

$x_{3}=\frac{p_{2}\left(\mu-x_{1}^{2}\right)+\lambda x_{2}\left(x_{1}-\mu\right)-2 p_{2} x_{2}^{2}}{-2 p_{2}\left(\mu+x_{1}\right)}$,

if $\mu+x_{1} \neq 0$. Several observations immediately follow:

- $\mu+x_{1} \neq 0$ and $x_{3}$ does not lie on the surface (58), then the determinant (57) is nonzero, the CRC holds and the system (37) is weakly controllable.

- If $\mu+x_{1}=0$, i.e. $x_{1}=-\mu$, then the determinant (57) becomes

$\operatorname{det}\left(\left[\vec{g}_{3},\left[\vec{f}_{3}, \vec{g}_{3}\right],\left[\vec{f}_{3},\left[\vec{f}_{3}, \vec{g}_{3}\right]\right]\right]\right)=8 \lambda \mu p_{2} x_{2}\left(\lambda \mu+p_{2} x_{2}\right)$,

which is not zero when $x_{2} \neq 0$ and $x_{2} \neq-\lambda \mu / p_{2}$. Then the CRC holds and the system (37) is weakly controllable.

To summarize, consider three surfaces:

$$
\begin{aligned}
& S_{8}=\left\{\left(x_{1}, x_{2}, x_{3}, p_{1}, p_{2}\right) \mid \mu+x_{1}=0\right\}, \quad S_{9}=\left\{\left(x_{1}, x_{2}, x_{3}, p_{1}, p_{2}\right) \mid x_{3}=\frac{p_{2}\left(\mu-x_{1}^{2}\right)+\lambda x_{2}\left(x_{1}-\mu\right)-2 p_{2} x_{2}^{2}}{-2 p_{2}\left(\mu+x_{1}\right)}\right\}, \\
& S_{10}=\left\{\left(x_{1}, x_{2}, x_{3}, p_{1}, p_{2}\right) \mid x_{2}=0 \text { or } \lambda \mu+p_{2} x_{2}=0\right\}
\end{aligned}
$$

in the state-parameter space $R^{5}=\left\{\left(x_{1}, x_{2}, x_{3}, p_{1}, p_{2}\right)\right\}$. Then the system is weakly controllable at all points in $R^{5} \backslash\left\{S_{8} \cup S_{9}\right\}$. In $S_{8}$, the system is weakly controllable at all points in $S_{8} \backslash S_{10}$.

\section{Some remarks on 3D models}

It should be pointed out that we study the 2D fluids mainly due to their mathematical convenience. In fact, if we assume that the stress tensor is of the following form

$$
\mathbf{T}=\left[\begin{array}{ccc}
T_{11} & T_{12} & 0 \\
T_{12} & T_{22} & 0 \\
0 & 0 & 0
\end{array}\right],
$$

then the 3D UCM model is reduced to the 2D model considered in this paper. If one consider the full 3D UCM model coupled with a three-dimensional flow, then the analysis will be much more complicated. As an example, consider the 3D UCM model in the presence of a 3D elongational flow where the velocity field is described by $\mathbf{v}=(-\dot{\gamma}(t)(x / 2)-\dot{\gamma}(t)(y / 2), \dot{\gamma}(t) z)$ and the UCM model (3) can be expressed as

$$
\frac{\mathrm{d} \vec{x}}{\mathrm{~d} t}=\vec{f}(\vec{x})+\vec{g}(\vec{x}) u
$$

where

$$
\vec{x}=\left[\begin{array}{l}
x_{1} \\
x_{2} \\
x_{3} \\
x_{4} \\
x_{5} \\
x_{6}
\end{array}\right]=\left[\begin{array}{l}
T_{11} \\
T_{12} \\
T_{13} \\
T_{22} \\
T_{23} \\
T_{33}
\end{array}\right], \quad u=\dot{\gamma}(t), \quad \vec{f}(\vec{x})=-\lambda \vec{x}, \quad \vec{g}(\vec{x})=\left[\begin{array}{c}
\mu-x_{1} \\
-x_{2} \\
\frac{1}{2} x_{3} \\
-\mu-x_{4} \\
\frac{1}{2} x_{5} \\
2 \mu+2 x_{6}
\end{array}\right] .
$$

The Lie brackets are

$$
[\vec{f}, \vec{g}]=\left[\begin{array}{c}
-\lambda \mu \\
0 \\
0 \\
\lambda \mu \\
0 \\
2 \lambda \mu
\end{array}\right] \equiv \vec{g}_{1}, \quad\left[\vec{f}, \vec{g}_{1}\right]=\lambda \vec{g} \equiv \vec{g}_{2}, \cdots
$$


It is obvious that the determinant of the Lie brackets vanishes and one cannot conclude weak controllability in 3D case. However, it is always possible to find control invariant submanifolds in the state space that are weakly controllable. The characterization of such invariant submanifold needs geometric analysis [2] which is beyond the scope of this paper.

Generally speaking, for 3D models coupled with 3D flows, the controllability becomes more subtle and complicated. One needs to use geometric approach to identify controllable submanifolds.

\section{Concluding remarks}

In this paper we have applied the controllability rank condition to the vector fields in the upper convected Maxwell model to study the controllability of viscoelastic fluids driven by various homogeneous flow fields. In our control system, the state variable is the stress and the available control is related to different flow rate. For the upper convected Maxwell model coupled with different flow fields, we find that

- The UCM model under extensional flow is weakly controllable on a stable invariant submanifold when the first normal stress difference $T_{11}$ does not equal to the second normal stress difference $T_{22}$. The reachable set is also derived.

- The UCM model under shear flow is weakly controllable on a stable invariant submanifold when the shear stress $T_{12}$ is nonzero.

- The UCM model under general planar linear flow is weakly controllable in some subsets of the state space. The constraints that define these subsets are characterized explicitly by equations of the stress components.

\section{Acknowledgments}

This research was supported in part by the Air Force Office of Scientific Research grant F1ATA06313G003, the Army Research Office and the National Science Foundation. The authors thank the anonymous referees for their constructive and critical suggestions and comments in improving this manuscript.

\section{References}

[1] R.B. Bird, R.C. Armstrong, O. Hassager, Dynamics of Polymeric Liquids, 2nd edition, Wiley, 1987.

[2] A. Isidori, Nonlinear Control Systems, 3rd edition, Springer, 1995.

[3] R.G. Larson, The Structure and Rheology of Complex Fluids, Oxford, 1998.

[4] H. Nijmeijer, A.J. van der Schaft, Nonlinear Dynamical Control Systems, Springer, 1990.

[5] M. Renardy, Are viscoelastic flows under control or out of control? Syst. Control Lett. 54 (2005) 1183-1193.

[6] M. Renardy, Shear flow of viscoelastic fluids as a control problem, J. Non-Newton. Fluid Mech. 131 (2005) 59-63.

[7] M. Renardy, On control of shear flow of an upper convected Maxwell fluid, Z. Angew. Math. Mech. 87 (2007) 213-218. 